\title{
Buschke-Ollendorf Syndrome: Report of a Case and a Brief Molecular Overview
}

\author{
Michel van Geel ${ }^{1,3}$, Valerie L.R.M. Verstraeten ${ }^{1,3}$, G.P.H. Lucker ${ }^{2}$ and Maurice A.M. van Steensel ${ }^{* 1,13}$ \\ ${ }^{I}$ Department of Dermatology, University Hospital Maastricht, Maastricht, The Netherlands \\ ${ }^{2}$ Atrium Hospital Heerlen, The Netherlands \\ ${ }^{3}$ GROW research institute, University of Maastricht, The Netherlands
}

\begin{abstract}
Buschke-Ollendorf syndrome (BOS) is a rare autosomal dominant disorder characterized by localized increases in bone density manifesting as osteopoikilosis or melorheostosis and connective tissue nevi, collagenomas. Manifestations are highly variable. It is caused by loss-of-function mutations in the LEMD3 gene, which codes for an inner nuclear membrane protein that is also known as MAN1. Six different mutations have been described to date without a clear genotype-phenotype correlation. Buschke-Ollendorf syndrome exemplifies the importance of TGFß signaling for bone and connective tissue homeostasis. Here, we report on a father and his daughter with typical BOS syndrome caused by a known nonsense mutation and provide an overview of what is now known of this rare disorder.
\end{abstract}

Keywords: Buschke-Ollendorf, MAN1, LEMD3, nuclear envelope, TGF-beta.

\section{INTRODUCTION}

Buschke-Ollendorf syndrome (BOS, MIM \#166700) is characterized by the occurrence from early childhood of disseminated connective tissue nevi and circumscribed sclerotic areas near the ends of many bones known as osteopoikilosis [1]. The latter can also manifest as linear sclerotic bone lesions with fibrosis of the overlying soft tissues, a condition known as melorheostosis [2]. BOS was first described in 1915 by Albers-Schönberg. Buschke and Helen Ollendorff reported it in 1928 (R. Happle, personal communication, 2007).

BOS is caused by heterozygous loss of function mutations in the gene coding for the inner nuclear membrane protein MAN1, also known as LEMD3 because it contains a LEM-domain [3]. Mutations have also been described in some, but not all, cases of melorheostosis, suggesting that this condition may be genetically heterogeneous (G. Mortier, personal communication, 2007).

LEMD3 interacts with BMP and activin-TGFß receptor activated Smads [4] and antagonizes both signaling pathways. This observation is consistent with earlier results from our group that show deregulation of TGF $\beta$ signaling in the context of nuclear envelope dysfunction [5]. Thus, BOS might be considered as a disruption of connective tissue homeostasis. All mutations observed so far have been truncating ones (nonsense, insertions and deletions) and a genotypephenotype correlation has so far not been demonstrated. To illustrate the clinical phenotype, we describe a father and his daughter with classical manifestions of BOS caused by a nonsense mutation in exon 1 of the LEMD3 gene.

*Address correspondence to this author at the Maastricht University Center for Molecular Dermatology, Department of Dermatology, University Hospital Maastricht, P.O. Box 5800, 6202AZ Maastricht, The Netherlands; Tel: +31433871641; E-mail: mvst@sder.azm.nl

\section{CASE REPORT}

The proposita, a 7-year old girl of Dutch descent, visited our outpatient clinic for evaluation of painless skin nodules on the lower back that had been present from the first year of life onwards. The lesions were slowly progressive. No other abnormalities had been noted. Her father noted that he had similar skin lesions on the medial sides of both knees, but had never paid much attention to them.

Upon examination, we saw a healthy-looking girl with no obvious dysmorphic traits. Hair, nails and teeth were normal. On the mid-thoracic and lumbar back we observed multiple partly confluent skin-colored nodules that were rather soft to palpation (Fig. 1). We noted no other skin abnormalities. Examination of the father revealed similar skin lesions on the medial sides of both knees (Fig. 2). Thinking of Buschke-Ollendorf syndrome, we acquired roentgenographs of the father's femur and pelvis. These showed clear metaphyseal osteopoikilosis.

Having confirmed the diagnosis, we performed mutation analysis of the $L E M D 3$ gene in both patients. Upon obtaining informed consent from the father, we isolated DNA from peripheral blood leucocytes using standard salt-precipitation methods as described previously [6]. Next, we amplified with PCR the coding regions and intron-exon boundaries of the LEMD3 gene. PCR products were subjected to direct sequencing using the BigDyeDeoxyTerminator system on an ABI 3100 capillary sequencer (Applied Biosystems, Warrington, UK). Primer sequences are listed in Table 1. Reaction conditions for PCR and sequencing are the same for all primer sets and are as follows: initial denaturation at $94^{\circ} \mathrm{C}$ for 90 seconds followed by 30 cycles of 30 seconds at $94{ }^{\circ} \mathrm{C}$, 30 seconds at $62{ }^{\circ} \mathrm{C}, 60$ seconds at $72{ }^{\circ} \mathrm{C}$ and termination at $72{ }^{\circ} \mathrm{C}$ for 7 minutes. We append M13 sequencing tails to our primers and use those to sequence. In both patients, we found a transversion $1322 \mathrm{C}>\mathrm{A}$ in the $L E M D 3$ gene leading to the truncating mutation $\mathrm{Y} 441 \mathrm{X}$ that was previously described by Hellemans et al. [7]. 

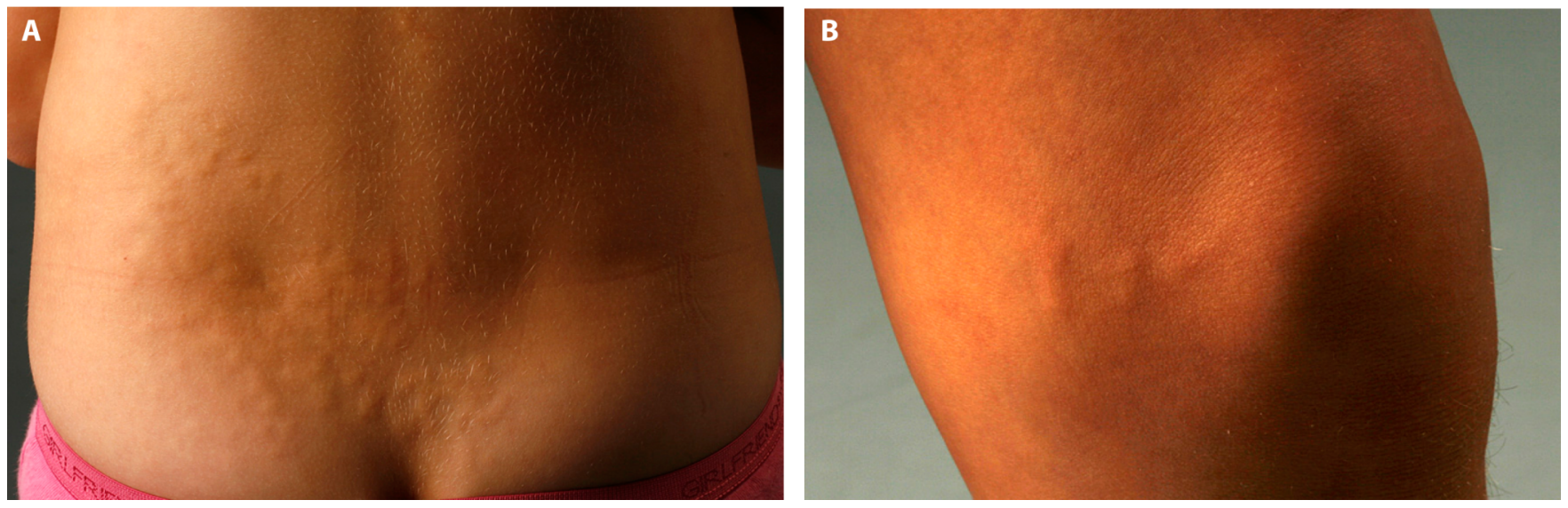

Fig. (1). The proposita. Subcutaneous aggregated nodules on the lower back. N. medial side of the father's left knee.

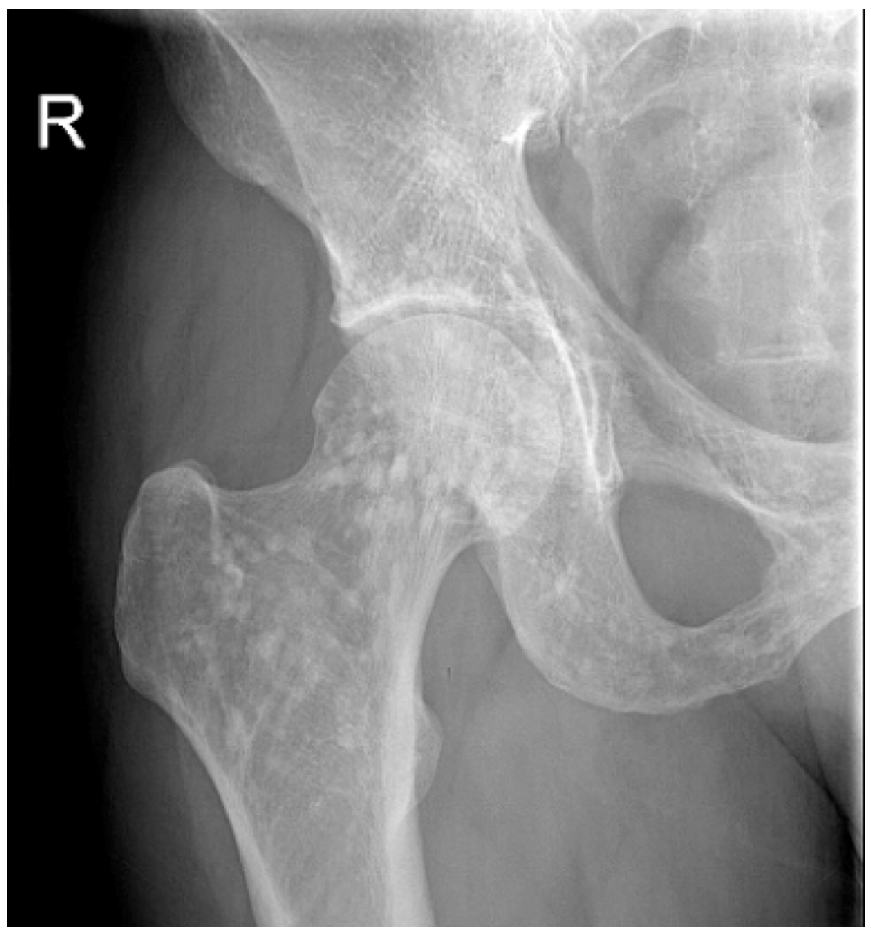

Fig. (2). Pelvic roentgenograph of the father showing osteopoikilosis.

\section{DISCUSSION}

We found a known nonsense mutation in the LEMD3 gene, causing classical Buschke-Ollendorf syndrome in a father and his daughter. All mutations described so far are likewise predicted to truncate the protein, causing a loss of function. The available functional data indicate that LEMD3 is involved in regulation of BMP- and TGFß-receptor mediated signaling. Specifically, LEMD3 can reduce the capacity of BMP4 to upregulate Smad6, Smad7, Id2 and Id3 [8]. Moreover, LEMD3 can also inhibit TGFß signaling [9]. The inhibition likely takes place through interactions between the LEMD3 C-terminus and the Smad MH2 domains. All known mutations, including the one we describe here, are expected to disrupt this interaction. Both the bone abnormalities and the connective tissue nevi are thought to result from dysregulation of TGFß signaling. In this respect, it is of interest to note that collagenomas can also occur in tuberous sclerosis
Table 1. Primer Sequences (M13 Tails Not Shown for Clarity)

\begin{tabular}{|l|l|}
\hline \multicolumn{1}{|c|}{ Primer Name } & \multicolumn{1}{c|}{ Sequence 5' -> ' } \\
\hline \hline LEMD3F1 & CGGTAGCGCGGAGCTTGTAA \\
\hline LEMD3R1c & GTTAGTCACCTCGCTGGCGG \\
\hline LEMD3F1a & GGTCTCGGGCGACCTCTCCT \\
\hline LEMD3R1a & CTATGGGTTCGTCTGGGCCG \\
\hline LEMD3F1b & CTGCAGAGCGAAGGAAGCCC \\
\hline LEMD3R1b & CGCACTGGGAGGGAGACTGT \\
\hline LEMD3F1c & CCCCCGCCACTTACTGACAT \\
\hline LEMD3R1 & ATGCACGCACTGTTGCGTTT \\
\hline LEMD3F2 & CACCAGTTTGTTTACATTTGGTTCACA \\
\hline LEMD3R2 & CCAACAACTACAGGCAACAGGCA \\
\hline LEMD3F3 & GGGATTGGGAACATTGCTTTGG \\
\hline LEMD3R3 & CCTCTAATACAGATGGCAGGCAGGA \\
\hline LEMD3F4 & CTGGAGGGCGTCTTGTGTGC \\
\hline LEMD3R4 & ACCAAACAGCAGGCCCAAGC \\
\hline LEMD3F5 & AAAGGATACTTTACAGAGAGTCGAATG \\
\hline LEMD3R6 & TGTGACTTATGTGGCAACCATC \\
\hline LEMD3F7 & GCTAATTCAGCCATCTGTCTTGAAGG \\
\hline LEMD3R8 & GCAAGTCTAGTTGAGAAGGGTCACAGC \\
\hline LEMD3F9 & TCCTGAAGCAGCATCTTGACCC \\
\hline LEMD3R9 & TGATTCCTTCTACGAAACAGAACGAGA \\
\hline LEMD3F10 & CCTTTCAACAAACTAGAACAATGTCAA \\
\hline LEMD3R10 & TTGGCCAAATCTTTGCTTGGA \\
\hline LEMD3F11 & GGAAAGTGGGAGGAGGGCTG \\
\hline LEMD3R12 & TGGTAAAAGACATATGAGCACAAAACA \\
\hline LEMD3F12 & GCATTGCATGGCTCTTGGTTTG \\
\hline
\end{tabular}

(TSC). The connection between TSC and BOS may not be immediately obvious, until one realizes that the TSC1/2 complex is a negative regulator of mTOR, mammalian target 


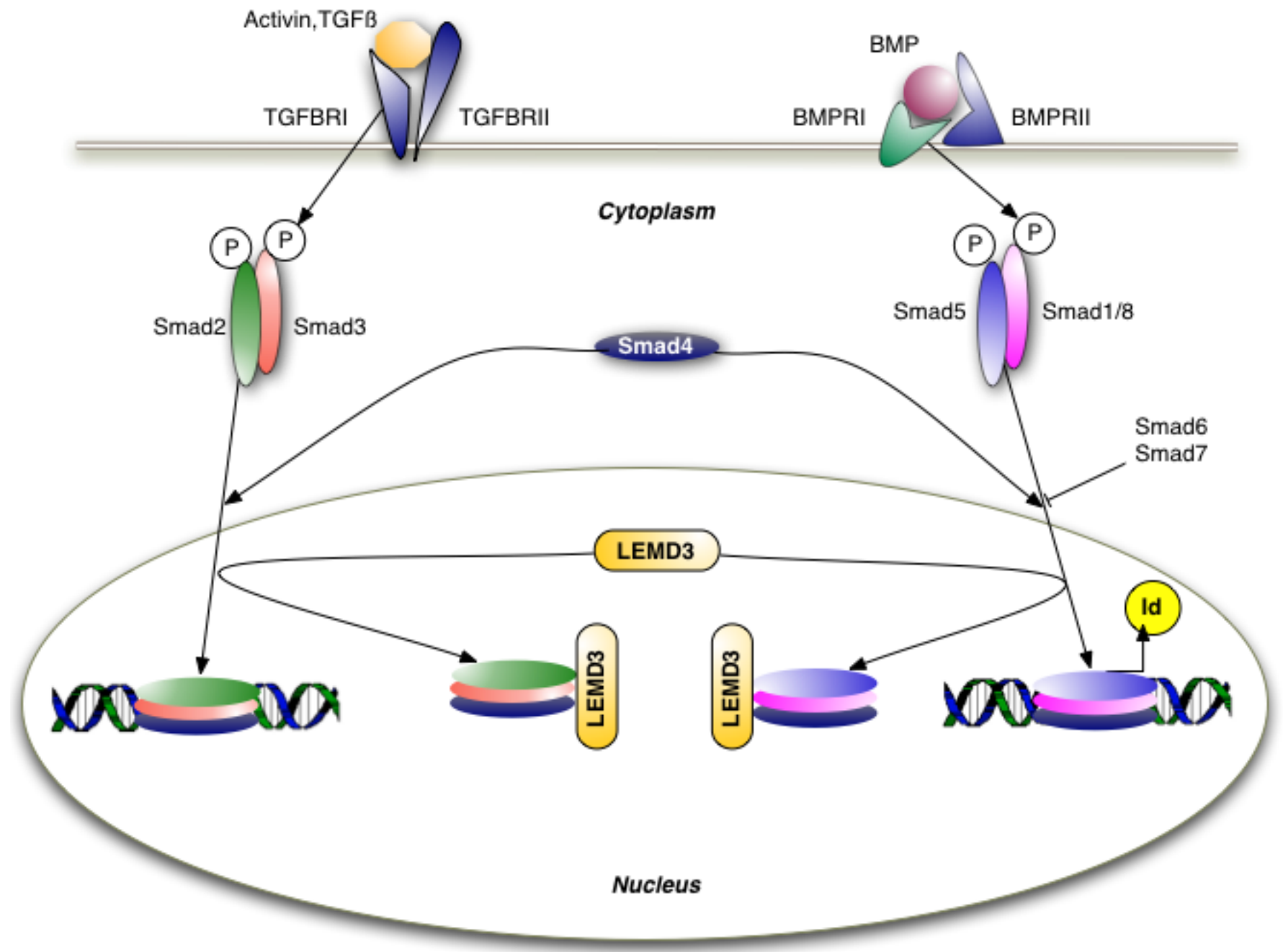

Fig. (3). Activin/TGFß/BMP signaling cascade. Activin/TGFß and BMPs signal through their respective receptor complexes to Smads that multimerize and act as transcription factors, regulating expression of among others, Smad family members (such as 6 and 7) and Id proteins. LEMD3 can sequester Smad complexes, preventing them from binding to their target sequences. P: phosphate group added by receptor complex. TGFß: transforming growth factor beta, BMP: bone morphogenetic proteins, Smad: suppressor of mothers against decapentaplegic, LEMD3: LEM domain containing 3 (aka MAN1), Id: inhibitor of differentiation.

of rapamycin [10]. A central regulator of cellular energy metabolism, mTOR seems to be directly involved in modulating Smad activity. For instance, rapamycin can induce Smad activity in prostate cancer cells [11]. Thus, dysregulation of mTOR can interfere with TGFß signaling, which possibly explains the collagenomas found in TSC.

Nosologically, BOS belongs to a family of connective tissue diseases characterized by abnornalities of TGFß signaling that affect bone density. For example, sclerosteosis (OMIM 269500) is caused by loss of fucntion of the SOST gene coding for the BMP antagonist sclerostin [12]. Camurati-Engelman disease, which is caused by activating mutations in the TGFB1 gene [13-15], has some resemblance to BOS in that it is characterized by chronic thickening of the metaphyses. Considering that both disorders feature increased TGFß-signaling, this is not surprising. However, bone symptoms in Camurati-Engelmann syndrome are considerably more severe and the disorder will incapacitate patients unless treated with corticosteroids [16]. Skin abnormalities, on the other hand, are not found. This is rather un- expected, because sclerotic skin changes can be observed in individuals with melorheostosis [17]. Moreover, increased TGFß signaling has also been reported in scleroderma [18] and is partly responsible for the stiff skin syndrome phenotype (B. Loeys, personal communication, 2007). Sclerodermiform skin disease is furthermore found in progeroid laminopathies caused by mutations in the $L M N A$ gene or in ZMPSTE24, which codes for an enzyme that is required for one of the final steps in lamin A protein post-translational processing [19]. It was recently shown that A-type lamins are required for the regulation of TGF- $\$ 1$ mediated collagen production [5]. Lamin A can bind to LEMD3 through a distinct domain located towards the C-terminus [20]. The LEM domain binds to the DNA-binding protein BAF and LEMD3 can thus act as a bridge between the nuclear lamina and DNA. It is tempting to speculate that these interactions are required for proper suppression of Smad signaling and may be specific to the LEM domain, as closely related LEM domain proteins also appear to be involved in Smad interactions [21]. Disruption of binding to either lamin or BAF might explain part of the sclerosis observed in laminopathies 
and BOS alike. However, no mutation described to date specifically affects either the lamin binding or LEM domains, rendering this explanation unlikely. Hellemans et al. described a patient with a microdeletion encompassing the LEMD3 gene [22]. While he had an extended phenotype with osteopoikilosis, his deletion affected several contigous genes. Thus it is not possible to attribute his more extensive phenotype to absence of the lamin binding and LEM domains. In conclusion, a genotype-phenotype correlation does not emerge from the known mutational spectrum. Hence, the clinical variability of BOS may be caused by individual variations in TGFß and/or BMP signaling.

\section{ACKNOWLEDGEMENTS}

$\mathrm{MvG}$ and MvS are supported by grants from the University Hospital Maastricht, Stichting Drie Lichten, ZONMW grant number 907-00-202 and Barrier Therapeutics, NV.

VV is supported by the University Hospital Maastricht and a ZONMW Horizon grant.

\section{REFERENCES}

[1] Kim GH, Dy LC, Caldemeyer KS, Mirowski GW. BuschkeOllendorff syndrome. J Am Acad Dermatol 2003; 48(4): 600-1.

[2] Greenspan A, Azouz EM. Bone dysplasia series. Melorheostosis: review and update. Can Assoc Radiol J 1999; 50(5): 324-30.

[3] Hellemans J, Preobrazhenska O, Willaert A, et al. Loss-of-function mutations in LEMD3 result in osteopoikilosis, Buschke-Ollendorff syndrome and melorheostosis. Nat Genet 2004; 36(11): 1213-8.

[4] Lin F, Morrison JM, Wu W, Worman HJ. MAN1, an integral protein of the inner nuclear membrane, binds Smad2 and Smad3 and antagonizes transforming growth factor-beta signaling. Hum Mol Genet 2005; 14(3): 437-45.

[5] Van Berlo JH, Voncken JW, Kubben N, et al. A-type lamins are essential for TGF-beta1 induced PP2A to dephosphorylate transcription factors. Hum Mol Genet 2005; 14(19): 2839-49.

[6] Miller SA, Dykes DD, Polesky HF. A simple salting out procedure for extracting DNA from human nucleated cells. Nucleic Acids Res 1988; 16(3): 1215.

[7] Hellemans J, Debeer P, Wright M, et al. Germline LEMD3 mutations are rare in sporadic patients with isolated melorheostosis. Hum Mutat 2006; 27(3): 290.

[8] Osada S, Ohmori SY, Taira M. XMAN1, an inner nuclear membrane protein, antagonizes BMP signaling by interacting with Smad1 in Xenopus embryos. Development 2003; 130(9): 1783-94.
Ishimura A, Ng JK, Taira M, Young SG, Osada S. Man1, an inner nuclear membrane protein, regulates vascular remodeling by modulating transforming growth factor beta signaling. Development 2006; 133(19): 3919-28.

[10] Sarbassov dos D, Ali SM, Sabatini DM. Growing roles for the mTOR pathway. Curr Opin Cell Biol 2005; 17(6): 596-603.

[11] van der Poel HG, Hanrahan C, Zhong H, Simons JW. Rapamycin induces Smad activity in prostate cancer cell lines. Urol Res 2003; 30(6): 380-6

[12] Brunkow ME, Gardner JC, Van Ness J, et al. Bone dysplasia sclerosteosis results from loss of the SOST gene product, a novel cystine knot-containing protein. Am J Hum Genet 2001; 68(3): 57789.

[13] Saito T, Kinoshita A, Yoshiura K, et al. Domain-specific mutations of a transforming growth factor (TGF)-beta 1 latency-associated peptide cause Camurati-Engelmann disease because of the formation of a constitutively active form of TGF-beta 1. J Biol Chem 2001; 276(15): 11469-72.

[14] Janssens K, Gershoni-Baruch R, Guanabens N, et al. Mutations in the gene encoding the latency-associated peptide of TGF-beta 1 cause Camurati-Engelmann disease. Nat Genet 2000; 26(3): 273-5.

[15] Kinoshita A, Saito T, Tomita H, et al. Domain-specific mutations in TGFB1 result in Camurati-Engelmann disease. Nat Genet 2000; 26(1): $19-20$

[16] Janssens K, Vanhoenacker $\mathrm{F}$, Bonduelle $\mathrm{M}$, et al. CamuratiEngelmann disease: review of the clinical, radiological, and molecular data of 24 families and implications for diagnosis and treatment. J Med Genet 2006; 43(1): 1-11.

[17] Wagers L, Young A, Ryan S. Linear melorheostotic scleroderma. Br J Dermatol 1972; 86(3): 297-301.

[18] Verrecchia F, Mauviel A, Farge D. Transforming growth factorbeta signaling through the Smad proteins: role in systemic sclerosis. Autoimmun Rev 2006; 5(8): 563-9.

[19] Sevenants L, Wouters C, De Sandre-Giovannoli A, et al. Tight skin and limited joint movements as early presentation of HutchinsonGilford progeria in a 7-week-old infant. Eur J Pediatr 2005; 164(5): 283-6.

[20] Gruenbaum Y, Margalit A, Goldman RD, Shumaker DK, Wilson KL. The nuclear lamina comes of age. Nat Rev Mol Cell Biol 2005; 6(1): 21-31.

[21] Raju GP, Dimova N, Klein PS, Huang HC. SANE, a novel LEM domain protein, regulates bone morphogenetic protein signaling through interaction with Smad1. J Biol Chem 2003; 278(1): 42837.

[22] Menten B, Buysse K, Zahir F, et al. Osteopoikilosis, short stature and mental retardation as key features of a new microdeletion syndrome on 12q14. J Med Genet 2007; 44(4): 264-8. 